\title{
Escolha de modo no acesso terrestre a aeroportos considerando a confiabilidade do tempo de viagem
}

\author{
Bianca Bianchi Alves ${ }^{1}$ e Orlando Strambi ${ }^{2}$
}

\begin{abstract}
Resumo: O objetivo do trabalho é identificar variações sistemáticas da importância atribuída por viajantes aéreos aos fatores que influenciam a escolha de modo para acesso terrestre ao Aeroporto Internacional de São Paulo. A análise utilizou dados de uma pesquisa de preferência declarada conduzida com residentes na região viajando para destinos internacionais. Os indivíduos ordenavam 4 alternativas: automóvel, táxi, ônibus expresso existente e um trem expresso proposto, descritas pelos atributos de custo, tempo esperado de viagem e confiabilidade do tempo de viagem, expressa através de uma margem de segurança. Modelos de escolha discreta foram estimados, considerando os efeitos de painel, aninhamento e de inércia. Os resultados indicaram que viajantes a negócios e de alta renda são menos sensíveis ao custo da viagem de acesso ao aeroporto. A importância da confiabilidade do tempo de viagem é maior do que a atribuída ao tempo esperado de viagem e não foi afetada por variações sistemáticas.
\end{abstract}

Palavras-chave: valor de confiabilidade; acesso terrestre a aeroportos; escolha de modo; modelo logit misto; preferência declarada.

\begin{abstract}
The paper analyses systematic variation of tastes and preferences of air travelers regarding the choice of mode for the ground access to the São Paulo International Airport. A stated preference survey was conducted with individuals traveling to international destinations. Each respondent ranked 4 alternatives: auto, taxi, the existing express bus, and a proposed express train. Attributes describing modes were travel cost, average travel time and travel time reliability, expressed as a safety margin - the time period allocated by the individual for arriving at the airport at the preferred time. Discrete mode choice models were estimated, considering panel, nesting and inertia effects. Results indicated that business and higher income travelers are less sensitive to access cost. The importance of the reliability of travel time is higher than the importance of average travel time and is not affected by systematic variations.
\end{abstract}

Keywords: value of realibility; ground access to airport; modal choice; mixed logit model; stated preference.

\section{INTRODUÇÃO}

$\mathrm{O}$ acesso terrestre a aeroportos em grandes áreas urbanas pode ser seriamente afetado pelo congestionamento de tráfego urbano; em alguns casos, o tempo de acesso pode ser maior do que o dedicado à viagem aérea propriamente dita. A provisão de acesso direto, muitas vezes por modo ferroviário, é uma importante alternativa considerada nestes casos. O estudo de viabilidade para este tipo de investimento requer estimar receitas de operação e, portanto, enfrenta as dificuldades de se prever a demanda atraída por novos modos de transporte.

Para prever a demanda é comum a utilização de modelos que consideram atributos das alternativas de transporte que influenciam o comportamento dos usuários. Usualmente, os modelos restringem-se a representar tempos e custos de viagem, variáveis mais facilmente mensuráveis. Reconhecese, porém, que outros fatores afetam, alguns deles de forma importante, o comportamento da demanda. Entre estes, destaca-se a confiabilidade do tempo de viagem, associada à variabilidade presente nos deslocamentos, geralmente em conseqüência da presença de congestionamentos.

A escolha de modo no acesso terrestre a aeroportos vem sendo estudada na literatura recente como um tópico de interesse acadêmico e aplicação prática (Transportation Research Board, 2008; Tsamboulas e Nikoleris, 2008). Ao

\footnotetext{
${ }^{1}$ Bianca Bianchi Alves, Departamento de Engenharia de Transportes, Escola Politécnica, Universidade de São Paulo, São Paulo, SP, Brasil. (e-mail: biancaalves@gmail.com).

${ }^{2}$ Orlando Strambi, Departamento de Engenharia de Transportes, Escola Politécnica, Universidade de São Paulo, São Paulo, SP, Brasil. (e-mail: ostrambi@usp.br).
}

Manuscrito recebido em 20/6/2010 e aprovado para publicação em 25/2/2011. Este artigo é parte de TRANSPORTES v.19, n.1, 2011. ISSN: 2237-1346 (online). mesmo tempo, a pesquisa em confiabilidade do tempo de viagem vem apresentando progresso importante, na medida em que avanços conceituais e metodológicos são incorporados na análise (ITS, 2008; Vincent, 2008; Brownstone e Small, 2005). No entanto, são raros os estudos que consideram os dois temas simultaneamente - a confiabilidade do tempo de viagem no acesso a aeroportos - (Koster et al., 2010; Tam et al., 2008), apesar da importância deste atributo em uma situação na qual atrasos significativos implicariam altos custos.

O objetivo deste artigo é identificar variações sistemáticas da importância atribuída pelos viajantes aos fatores que afetam a escolha do modo de acesso ao aeroporto, inclusive a confiabilidade do tempo de viagem. O caso estudado é o do aeroporto internacional de São Paulo. Atualmente, o acesso de/para o centro de São Paulo é feito utilizando uma via expressa que apresenta elevados níveis de congestionamento; os tempos de viagem podem variar entre $40 \mathrm{mi}-$ nutos e mais de 2 horas para uma viagem de cerca de 25 $\mathrm{km}$, dependendo das condições de tráfego. Como motivação adicional, existem propostas para a construção de uma ligação expressa ferroviária ao aeroporto, que se distinguiria dos outros modos em virtude de uma esperada maior confiabilidade do tempo de viagem, por evitar o congestionamento comum aos modos rodoviários. Para identificar a importância dos fatores que afetam a escolha do modo, foi realizada uma pesquisa de preferência declarada - PD com viajantes partindo em vôos internacionais; os resultados foram utilizados para a estimação de modelos de escolha discreta de modo de transporte no acesso terrestre ao aeroporto.

$\mathrm{O}$ artigo está estruturado da seguinte maneira: o item 2 apresenta uma revisão de literatura sobre confiabilidade de tempo de viagem. O procedimento de coleta de dados é 
descrito no item 3 , enquanto no item 4 são feitas algumas análises preliminares dos dados obtidos. A especificação e estimação dos modelos e uma discussão dos resultados são feitas no item 5, seguindo-se as conclusões. Este artigo estende trabalho anterior dos autores (Alves e Strambi, 2010), que buscou identificar a estrutura de modelos que melhor descrevessem o comportamento observado.

\section{A CONFIABILIDADE DO TEMPO DE VIAGEM}

Bates et al. (2001), em sua revisão sobre confiabilidade do tempo de viagem, afirmam que o principal problema ao se estabelecer uma metodologia apropriada para quantificar a importância da confiabilidade é a dificuldade de encontrar maneiras de expressar a variabilidade do tempo de viagem (como percebido pelo indivíduo), ou seja, definir as variáveis a serem utilizadas na apresentação de estímulos em experimentos de PD e na estimação de modelos. Eles afirmam que a confiabilidade do tempo de viagem está associada com a noção estatística de variabilidade, que é a variância da distribuição, que por sua vez está relacionada ao tempo médio de viagem. Para os autores, todos os efeitos não originados exclusivamente da variabilidade - efeitos não aleatórios - devem ser descartados. Nesse sentido, variações decorrentes de congestionamento diário recorrente não devem ser incluídas na análise, porque eles não estão associados à variabilidade aleatória do tempo de viagem, mas às características específicas dos dias selecionados. Variações previsíveis, para esses autores, não são medidas da variabilidade do tempo de viagem.

No entanto, essa pode não ser a maneira como a variabilidade do tempo de viagem é compreendida pelos indivíduos. É difícil garantir que estes incluam em suas considerações apenas os efeitos aleatórios. Se a noção de confiabilidade relaciona-se à repetição e regularidade, há casos, como no acesso a aeroportos, nos quais as viagens não são frequentes. Mesmo que haja uma variação sistemática nos tempos de viagem, ela pode não ser conhecida ou percebida pelos indivíduos. De acordo com Noland e Polak (2002), há ainda grande incerteza quanto à capacidade de capturar de fato as percepções que as pessoas têm da variabilidade do tempo de viagem. Um recente e vasto estudo sobre o assunto conduzido pelo Institute for Transport Studies da Universidade de Leeds (ITS, 2008) indica a necessidade de pesquisa substancial sobre a valoração da variabilidade do tempo de viagem, investigando como a informação pode ser apresentada aos indivíduos e também como devem ser posteriormente especificados os modelos econométricos estimados com os dados coletados.

No presente estudo, uma característica importante delimita o conjunto de variáveis que podem ser utilizadas para indicar a variabilidade do tempo de viagem; no acesso terrestre a aeroportos, é comum que os viajantes agendem seu horário de saída para o aeroporto com suficiente antecedência, buscando garantir seu embarque no vôo desejado. As companhias de aviação geralmente sugerem que viajantes internacionais estejam no aeroporto com (pelo menos) duas horas de antecedência em relação à partida (a margem da companhia aérea para realizar o check-in e operações de despacho no aeroporto). Porém, a escolha do horário preferido de chegada ao aeroporto varia entre os viajantes e pode coincidir - ou não - com o sugerido pela companhia aérea (os viajantes mais frequentes podem se expor mais ao risco, por exemplo). Definido um horário preferido de chegada, podemos considerar que os viajantes decidem a sua hora de partida da origem (residência, trabalho, etc.) com base no tempo esperado de viagem e na variabilidade percebida do tempo de viagem. Eles adotam uma margem de segurança que os permita chegar ao aeroporto e proceder ao embarque com mais ou menos conforto, de acordo com a sua percepção.

Pells (1987) sugere que a margem de segurança é a forma como as pessoas reagem à variabilidade; portanto, seria a melhor maneira de medir a percepção de variabilidade. Para viagens a trabalho, o autor define a margem de segurança como a diferença entre o horário de chegada e o começo do turno de trabalho. Para Pells, forças contrárias estão agindo: o indivíduo tenta restringir atrasos a um nível aceitável, maximizando o tempo livre.

Em um experimento preferência declarada, além das incertezas que os viajantes têm que lidar neste tipo de viagem (infrequente e não familiar, requerendo uma margem de segurança no aeroporto), existe uma considerável quantidade de informação que os entrevistados devem processar além da percepção de confiabilidade. Eles têm que conhecer as alternativas disponíveis de transporte público (a presente pesquisa revelou que muitas vezes mesmo os residentes em São Paulo não tinham esse conhecimento) e selecionar hipoteticamente quais os terminais e modos de transporte até esses terminais eles escolheriam caso selecionassem determinada alternativa. Noland e Polak (2002) ressaltam que está presente uma complexidade adicional quando os viajantes têm que realizar uma transferência entre modos de transporte.

As razões acima levaram a adoção da margem de segurança, definida como o tempo reservado para a viagem terrestre para se chegar ao aeroporto no horário desejado, que pareceu de mais fácil compreensão pelos entrevistados, permitindo-os realizar escolhas hipotéticas. A margem de segurança é um conceito mais simples em relação aos enfoques baseados em programação (scheduling) propostos na literatura (Hollander, 2006), já que não explicita as considerações de hora de saída e preferida de chegada, assim como os custos por chegada adiantada ou atrasada.

\section{COLETA DE DADOS}

\subsection{População pesquisada e estrutura da pesquisa}

Em junho de 2005, dados de preferência revelada (PR) e declarada (PD) foram coletados de viajantes para destinos internacionais no Aeroporto Internacional de São Paulo. Entrevistas face-a-face utilizando laptops foram realizadas nos portões de embarque, com passageiros selecionados "aleatoriamente". As entrevistas foram aplicadas somente a residentes da Região Metropolitana de São Paulo buscando-se reduzir o viés gerado pela possível falta de informação sobre as alternativas disponíveis para o acesso ao aeroporto.

As entrevistas foram conduzidas em duas sextas-feiras e dois sábados. Esses dias apresentam características de acesso distintas; sexta-feira é em geral o dia da semana com as piores condições de tráfego, enquanto que aos sábados congestionamentos são menos frequentes.

As entrevistas foram realizadas em 3 estágios. No primeiro estágio, dados socioeconômicos e sobre a viagem aé- 
rea e terrestre foram levantados. No segundo estágio, o trem expresso proposto e o serviço de ônibus direto de alta qualidade foram apresentados. O conceito de margem de segurança foi também apresentado como detalhado abaixo. No terceiro estágio, o entrevistado ordenava alternativas de transporte segundo sua preferência, de acordo com o experimento descrito a seguir.

\subsection{Experimento de preferência declarada}

A coleta de dados PD vem se tornando a principal forma de coleta de dados sobre comportamento da demanda (Louviere et al., 2000). Enquanto modelos estimados com dados observados - dados de preferência revelada - PR - tendem a ser mais aderentes às escolhas realizadas pelos indivíduos, modelos PD apresentam mais estabilidade no tempo e no espaço. Dados PR são úteis para prever mudanças no equilíbrio de curto prazo, enquanto dados PD são ricos em informação sobre trade-offs entre características das alternativas e melhores para prever comportamento. Outra vantagem dos dados PD é que eles contêm mais informação por entrevistado, já que cada indivíduo provê múltiplas respostas em cenários de escolha ou ordenação de alternativas. Amostras de indivíduos em pesquisas PD tendem então a serem menores do que as necessárias usando dados PR para a construção de modelos.

A pesquisa foi baseada em um experimento fatorial ortogonal de escolha (Louviere et al., 2000). Três atributos foram considerados no acesso terrestre ao aeroporto - custo de viagem, tempo médio de acesso e margem de segurança do tempo de acesso - todos apresentados em três níveis. Os valores dos atributos para cada entrevista foram construídos a partir dos dados informados pelos indivíduos na pesquisa de preferência revelada, adicionando-se os valores apresentados na Tabela 1. O desenho foi elaborado de modo a eliminar situações dominantes de escolha (com os três atributos de uma alternativa melhores - ou piores - do que os das outras alternativas). Os questionários foram construídos para evitar fadiga e protocolos de decisão tais como omissão de restrições e outros vieses.

Tabela 1. Variação dos atributos

\begin{tabular}{lccc}
\hline Atributo & Nível 0 & Nível 1 & Nível 2 \\
\hline Tempo (min) & -10 & 0 & 20 \\
Custo (R\$) & -5 & 0 & 10 \\
Margem de segurança (min) & 10 & 20 & 30 \\
\hline
\end{tabular}

Cada indivíduo procedia à execução de três exercícios de ordenação (ranking) ou cenários, cada um deles contendo um conjunto de quatro alternativas, uma para cada modo considerado: carro, táxi, o serviço de ônibus direto existente (que parte de diversos terminais em São Paulo) e o novo trem expresso proposto, ligando o centro de São Paulo ao aeroporto. Para tornar o experimento mais realista, cenários individualizados de tempo e custo de viagem foram construídos utilizando a informação do bairro de origem da viagem terrestre, como informado pelo entrevistado. Para o ônibus e o trem, a necessidade de se estimar tempos e custos associados ao acesso ao terminal foi enfatizada e o passageiro deveria escolher um modo de transporte para essa etapa da viagem. Solicitou-se ainda aos entrevistados considerar que a viagem aérea seria realizada sem acompanhantes. Algumas telas da pesquisa são apresentadas na Figura 1.

\subsection{Apresentação do conceito da margem de segurança}

O conceito de margem de segurança foi apresentado ao entrevistado duas vezes ao longo da pesquisa. Em um primeiro estágio, o conceito foi introduzido e o respondente foi solicitado a responder à seguinte questão:

"No acesso terrestre ao aeroporto, normalmente enfrentamos congestionamento que afeta o nosso tempo de vi-

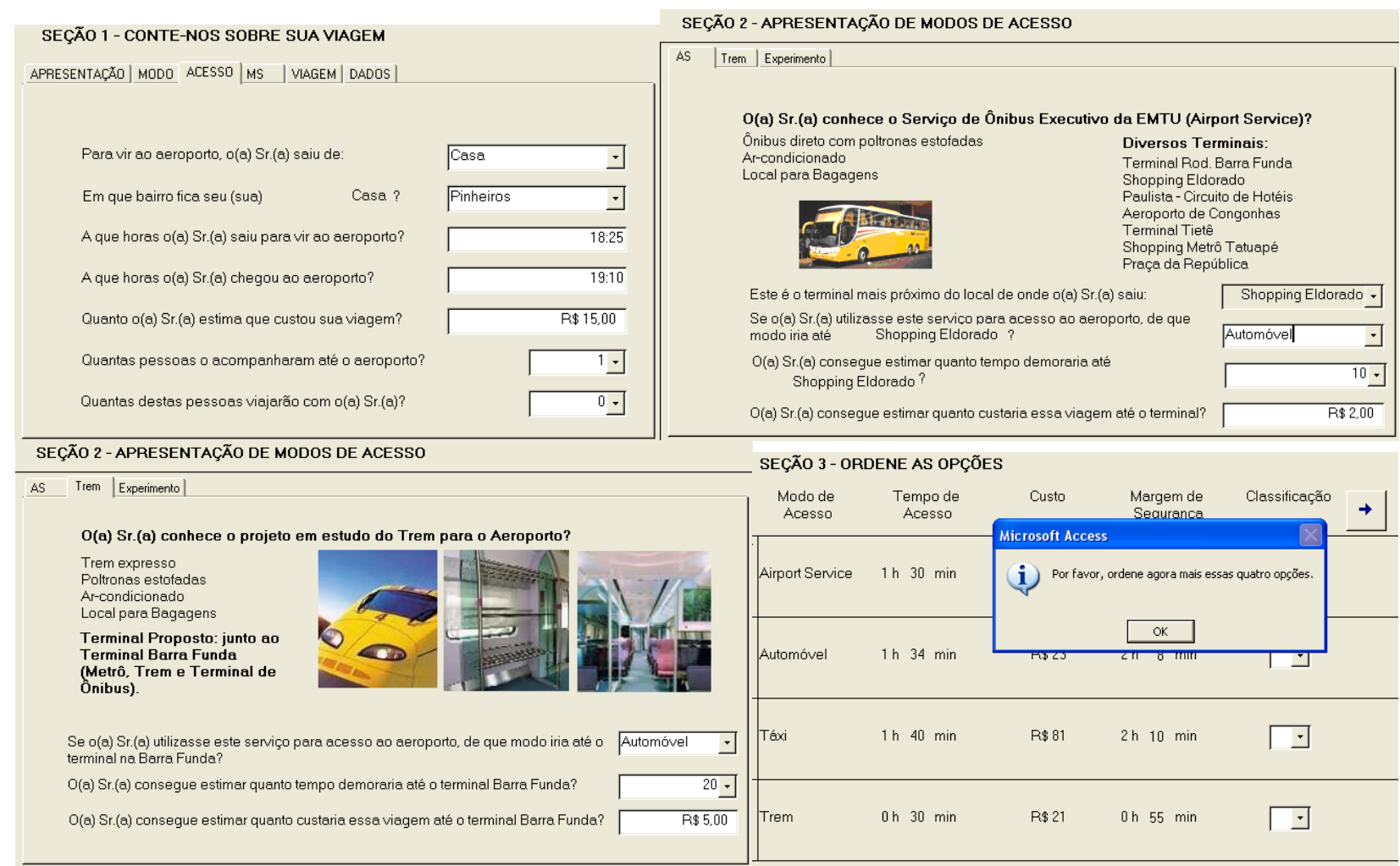

Figura 1. Telas da pesquisa 
Tabela 2. Distribuição dos indivíduos entrevistados por motivo, renda e dia da semana

\begin{tabular}{|c|c|c|c|c|c|c|}
\hline \multirow[b]{2}{*}{ Motivo } & \multicolumn{3}{|c|}{ Renda Individual Mensal } & \multicolumn{2}{|c|}{ Dia da Semana } & \multirow[t]{2}{*}{ Todos } \\
\hline & $\leq R \$ 4.000$ & $>R \$ 4.000$ & não informou & Sexta & Sábado & \\
\hline Negócios & $67 \%$ & $62 \%$ & $33 \%$ & $48 \%$ & $64 \%$ & $57 \%$ \\
\hline Lazer & $29 \%$ & $28 \%$ & $57 \%$ & $36 \%$ & $33 \%$ & $34 \%$ \\
\hline Ambos & $4 \%$ & $7 \%$ & $5 \%$ & $9 \%$ & $3 \%$ & $6 \%$ \\
\hline Outros & $0 \%$ & $3 \%$ & $5 \%$ & $7 \%$ & $0 \%$ & $3 \%$ \\
\hline Total & $23 \%$ & $57 \%$ & $20 \%$ & $42 \%$ & $58 \%$ & \\
\hline
\end{tabular}

Tabela 3. Distribuição dos modos de transporte no acesso terrestre

\begin{tabular}{lcccc}
\hline Modo de Transporte & \multicolumn{2}{c}{ Modo $^{\text {(*) }^{*}}$} & & Modo 2 \\
${$\cline { 1 - 3 }$} }$ & 62 & $(59 \%)$ & & 3 \\
Táxi & 36 & $(34 \%)$ & & 1 \\
Ônibus & 5 & $(5 \%)$ & & - \\
Outros & 2 & $(2 \%)$ & & - \\
Metrô & - & & & 1 \\
\hline Total & 105 & $(100 \%)$ & & 5 \\
\hline
\end{tabular}

$\left(^{*}\right)$ Modo 1 é o utilizado no último trecho no acesso ao aeroporto

Tabela 4. Resultados da ordenação feita pelos entrevistados no experimento PD

\begin{tabular}{|c|c|c|c|c|}
\hline Modo de transportes & $1^{a}$ opção & $2^{a}$ opção & $3^{a}$ opção & $4^{a}$ opção \\
\hline Carro & $35 \%$ & $26 \%$ & $23 \%$ & $16 \%$ \\
\hline Táxi & $11 \%$ & $27 \%$ & $20 \%$ & $42 \%$ \\
\hline Ônibus & $8 \%$ & $30 \%$ & $45 \%$ & $17 \%$ \\
\hline Trem & $46 \%$ & $17 \%$ & $12 \%$ & $25 \%$ \\
\hline
\end{tabular}

agem. Quanto tempo você reserva para sua viagem terrestre para chegar no horário desejado, considerando o modo de transporte utilizado?"

Mais tarde, na etapa do experimento PD, uma probabilidade foi associada ao conceito de margem de segurança:

"No primeiro estágio desta pesquisa, você respondeu quanto tempo reservava para chegar ao aeroporto no horário desejado. Você escolheu o que chamamos de uma margem de segurança"... "Com os valores de margem de segurança apresentados no próximo estágio, você pode considerar que chegará ao aeroporto no horário desejado em $90 \%$ das vezes."

É importante mencionar que os entrevistados pareceram compreender bem o conceito, como se escolher uma margem de segurança fosse um processo familiar. Além disso, eles pareceram confortáveis com a forma como a margem de segurança foi apresentada, com a hipótese de $10 \%$ de chance de chegar atrasado (um indicador de atitude face ao risco).

\section{ANÁLISE PREMILINAR DOS DADOS COLETADOS}

No total, 105 indivíduos foram entrevistados, sendo $39 \mathrm{mu}-$ lheres e 66 homens, com uma média de idade de 39 anos. A Tabela 2 indica que o motivo mais comum de viagem foi o de negócios (57\%), seguido de lazer (34\%); viagens que combinavam esses dois motivos representaram $7 \%$ da amostra e os 3\% remanescentes viajaram por outras razões. A renda declarada individual é superior a $\mathrm{R} \$ 4.000$ /mês para $57 \%$ dos entrevistados e inferior a este valor para $23 \%$ dos entrevistados; $20 \%$ optou por não declarar a renda. A tabela mostra ainda que viajantes a negócio eram mais frequentes nos sábados, o que deve ter ocorrido pela amostra ser composta apenas por viajantes internacionais.

A Tabela 3 apresenta os modos de transporte utilizados pelos entrevistados em seu acesso terrestre. O questionário permitia a combinação de até três modos para o acesso terrestre, mas apenas 5 pessoas utilizaram mais de um modo.

Os resultados obtidos no terceiro estágio da pesquisa, o exercício de ordenação $\mathrm{PD}$, indicaram forte preferência pelo trem, como demonstrado na Tabela 4 . Em 46\% dos cenários, o trem foi escolhido em primeiro lugar, superando os demais modos; porém, este foi também escolhido como última opção em $25 \%$ dos cenários. O carro foi a primeira escolha em $35 \%$ dos cenários; foi ainda o modo com menor índice de rejeição, sendo escolhido por último em apenas $16 \%$ dos cenários.

\subsection{Indivíduos com comportamento não compensatório}

Uma análise detalhada dos dados indicou que $75 \%$ dos entrevistados não mudaram a sua alternativa preferida nos três cenários. Duas categorias de indivíduos podem ser caracterizadas neste caso. A primeira inclui os entrevistados que escolheram como primeira opção na ordenação, nos três cenários de escolha, o modo de transporte utilizado no seu acesso terrestre naquele dia (modo da preferência revelada). Esses podem ser considerados cativos da sua escolha anterior, compondo uma categoria de indivíduos denominada de non-traders (Hess et al., 2010). Esse é um comportamento não compensatório, no qual a piora de um atributo não pode ser compensada pela melhora de outro. As funções de utilidade nos modelos de escolha discreta geralmente consideram comportamento compensatório e, portanto, recomenda-se excluir esses indivíduos da amostra. Havia 33 indivíduos nesta situação ( 25 cativos do carro) e eles foram excluídos da amostra, reduzindo-a a 72 indivíduos. Embora a retirada desses indivíduos impacte principalmente nas constantes específicas e coeficientes de inércia, exis- 
te a possibilidade que haja algum impacto nos coeficientes dos atributos do modelo (Hess et al., 2010).

A segunda categoria seria a de entrevistados que escolheram o trem como sua primeira opção em todos os cenários; estes não podem ser considerados cativos uma vez que esta não é atualmente uma opção existente. Esse resultado pode ter ocorrido devido às diferenças entre os níveis adotados para os atributos, que podem ter ficado abaixo do limiar de percepção do entrevistado, insuficiente para os indivíduos mudarem sua alternativa preferida nos três cenários. Variações maiores nos níveis, por outro lado, poderiam ter gerado alternativas não realistas. Outra possível explicação seria a presença de um viés de intenção política (policy bias), quando os indivíduos tentam influenciar a adoção de uma alternativa preferida. Não se pode descartar, porém, a hipótese de que este resultado reflita uma preferência extrema (no sentido proposto por Hess et al., 2010) pela alternativa trem, revelando a possível importância que seus atributos têm para o consumidor, em particular a variabilidade do tempo de viagem. Deve ser lembrado que o indivíduo era solicitado a considerar que a viagem era realizada desacompanhado, o que pode ter contribuído para aumentar a atratividade do trem. Por estas razões, esses indivíduos foram mantidos na amostra utilizada para a estimação dos modelos.

\subsection{Inconsistências na ordenação}

Exercícios de ordenação contêm múltiplas escolhas implícitas (pseudo-observações). No experimento aqui descrito pode-se dizer que a primeira escolha foi feita entre um conjunto de quatro alternativas, a segunda entre um conjunto de três alternativas (as restantes) e assim por diante. Nesse tipo de aplicação, porém, observa-se certa dificuldade em ordenar múltiplas alternativas. Em geral, os indivíduos claramente indentificam a melhor (e possivelmente a pior) alternativa, mas opções intermediárias são ordenadas com menor atenção.

Existem procedimentos relativamente complexos para lidar com este efeito, que pode ser representado admitindo-se diferentes variâncias para a variável dependente - a alternativa escolhida - dependendo do nível de ordenação (Ortúzar e Willumsen, 2001). Ben-Akiva et al. (1991) investigaram dados de ranking em uma análise empírica e afirmam que a confiabilidade dos dados descresce com a "profundidade" do rank; i.e., a primeira escolha é mais confiável que a segunda, e demais escolhas. Para Louviere et al. (2000) a questão central é definir se existe regularidade de preferências entre as diferentes fontes de dados.

A regularidade de preferências não foi verificada para os modelos estimados para cada uma das diversas profundidades dos ranks e a significância de modelos que incluíram escolhas de todas as profundidades foi menor do que modelos que incluíram apenas as primeiras escolhas. Com base nesses resultados, os dados para estimação dos modelos foram restritos às primeiras escolhas apenas; as respostas de ordenação foram substituídas por uma escolha simples entre quatro alternativas. Essa decisão resultou em uma redução dramática da informação disponível para estimação dos modelos. Além de um possível viés nas estimativas, essa redução dos dados traz importantes consequências na significância estatística dos parâmetros estimados. Os modelos estimados, especificações e resultados são discutidos a seguir.

\section{ESTIMAÇÃO DOS MODELOS DE ESCOLHA DISCRETA}

\subsection{Especificação dos modelos utilizados}

Modelos de escolha discreta foram estimados para avaliar a importância atribuída pelos indivíduos à confiabilidade do tempo de viagem. Nos últimos anos, os modelos do tipo logit misto (mixed logit) vêm sendo utilizados de forma crescente, em função de avanços teóricos e em capacidade de processamento. Esses modelos possuem uma estrutura mais flexível que os modelos logit multinomial, uma vez que não dependem das hipóteses de independência e homocedasticidade da parcela aleatória da utilidade das alternativas (Train, 2009). Modelos Logit Misto são integrais de modelos logit convencionais e seguem as seguintes fórmulas:

$$
\begin{gathered}
P_{n i}=\int L_{n i}(\beta) f(\beta) d \beta \\
L_{n i}(\beta)=\frac{e^{V_{n i}(\beta)}}{\sum_{j=1}^{J} e^{V_{n j}}(\beta)}
\end{gathered}
$$

em que,

$P_{n i}$ : probabilidade de escolha da alternativa $i$ para o indivíduo $n$;

$L_{n i}(\beta)$ : função de probabilidade logit para um dado valor do parâmetro $\beta$;

$V_{n i}(\beta)$ : parte observável da utilidade, que depende do parâmetro $\beta$; e

$f(\beta)$ : função de densidade para $\beta$.

Normalmente, admite-se $f(\beta)$ como uma função normal e estima-se a média e desvio padrão para $\beta$.

A partir de um conjunto de modelos logit misto desenvolvido por Alves e Strambi (2010), foram selecionados dois modelos que serviram de base para novas especificações que visaram verificar possíveis variações de preferências na população amostrada, melhorando a aderência dos modelos base aos dados e possivelmente representando de maneira mais fiel o comportamento de escolha. Em todos os casos, foram adotadas funções de utilidade linear, uma hipótese muito comum nas aplicações práticas (ITS, 2008).

Para representar nos modelos de escolha discreta variações de gostos ou preferências em uma população, pode-se proceder de duas maneiras: admitindo que as preferências são distribuídas aleatoriamente na população ou identificando variações sistemáticas de acordo com condições socioeconômicas dos indivíduos ou características de sua viagem. No primeiro caso, modelos logit misto de coeficientes aleatórios permitem que os coeficientes dos atributos das alternativas na função de utilidade variem na população segundo uma distribuição probabilística; os parâmetros destas distribuições (em geral valores esperados e variâncias) podem ser estimados. Embora algumas especificações de modelos mistos com coeficientes aleatórios tenham sido testadas, estas não mostraram superioridade estatística quando comparadas a modelos de coeficientes fixos.

Modelos com variações sistemáticas de preferência foram preferidos, utilizando-se variáveis binárias (dummy) para categorizar indivíduos na amostra. Essas variáveis binárias podem então interagir com os atributos (e com constantes específicas das alternativas), permitindo ao modelo capturar variações nos coeficientes (em relação à categoria 
de referência) (Ortúzar e Willumsen, 2001; Brito e Strambi, 2007). Os resultados permitem determinar se a diferença dos coeficientes entre as categorias é significativa. As categorias escolhidas podem considerar características dos indivíduos, como idade, gênero, renda, viajante frequente ou não, ou características da viagem, como motivo, dia da semana, etc.

\subsection{Descrição dos modelos base}

Com base nos dois melhores modelos obtidos por Alves e Strambi (2010) e utilizando o mesmo conjunto de dados, foram exploradas possíveis variações sistemáticas de preferências, objetivo deste trabalho. Naquele estudo, detectaram-se dois tipos de correlação importantes. Primeiro, a existência (esperada) do efeito painel - a correlação existente entre as respostas de um mesmo indivíduo, pela existência de fatores que são comuns às suas escolhas, tais como suas características socioeconômicas (Hensher e Greene, 2003). Modelos estimados com dados obtidos em pesquisas de preferência declarada, que coletam múltiplas respostas de cada indivíduo, devem incluir um termo que leve em conta o efeito painel. Não é possível representar adequadamente o efeito painel em modelos logit multinomial, sendo necessário utilizar uma especificação de logit misto. $\mathrm{O}$ modelo base I, descrito a seguir, considera o efeito painel. A segunda correlação importante foi verificada entre as alternativas de transporte coletivo (ônibus direto e trem expresso), possivelmente decorrente do fato desses modos compartilharem características importantes, como o desconforto/incerteza da espera em um terminal ou a falta de privacidade, não explicitamente consideradas na parcela observável da utilidade. A correlação entre alternativas pode ser representada em modelos logit hierárquicos (ou aninhados). Porém, esses modelos não permitem capturar o efeito painel, tornando-se necessário adotar uma estrutura que acomode os dois tipos de correlação observados, como o modelo base II apresentado abaixo.

O modelo base I é um modelo logit misto do tipo componentes de erro (error components) (Train, 2009). Apesar de formalmente iguais, o modelo de componentes de erro difere dos modelos logit misto de coeficientes aleatórios por incluir a aleatoriedade nos coeficientes específicos das alternativas, enquanto que nos modelos de coeficientes aleatórios são os coeficientes dos atributos que possuem uma distribuição probabilística. São chamados de componentes de erro porque o erro (a parte não observável da utilidade) é dividido em duas parcelas: uma parcela aleatória, que pode seguir qualquer distribuição, incluindo a normal, e uma parcela independente, identicamente distribuída como valor extremo (a mesma que dá origem aos modelos logit multinomial). $\mathrm{O}$ modelo base II é um modelo do tipo hierárquico (também chamado de generalizado de valor extremo, GEV, em inglês) misto (componentes de erro), que inclui em sua equação de utilidade termos que permitem representar os dois tipos de correlação: entre as alternativas (ninho) e entre respostas de um mesmo indivíduo (o efeito painel) (Train, 2009). O ninho considerado reuniu os modos de transporte coletivo (trem e ônibus).

Foi também incluída na especificação dos modelos uma variável representativa do efeito de hábito ou inércia, que leva o indivíduo a preferir alternativas conhecidas. Nesses casos, o comportamento é considerado como compensatório, mas a atratividade das alternativas menos utilizadas ou conhecidas tem que ser suficientemente grande para superar o efeito do hábito (Ortúzar e Willumsen, 2001). No caso de viagens não frequentes, como ao aeroporto, existem ainda menos oportunidades de se conhecer os atributos de alternativas nunca ou raramente utilizadas, o que pode reforçar o efeito de inércia na escolha do modo de acesso. Esse aspecto do comportamento pode ser capturado nos modelos de escolha discreta introduzindo uma variável que represente a escolha prévia do indivíduo em uma situação semelhante. Neste estudo, estava disponível a informação sobre o modo utilizado pelo entrevistado na viagem de acesso ao aeroporto (PR). Foi introduzida um variável binária nas funções de utilidade, recebendo o valor 1 no caso do modo utilizado no acesso, e zero nas funções de utilidade dos modos restantes. No caso do modelo base II, essa variável foi excluída da especificação final por não ser significante e, principalmente, apresentar sinal contrário ao esperado.

\subsection{Resultados dos modelos estimados}

Os modelos foram estimados com o software Biogeme (Bierlaire, 2008). A Tabela 5 apresenta os resultados da estimação dos modelos base e demais modelos que buscaram capturar variações sistemáticas de preferências. Analisando inicialmente os modelos base observa-se que o modelo base II apresenta melhor aderência aos dados do que o modelo base I ( $\rho^{2}$ ajustado $=0,388$ e 0,371 , respectivamente); decidiu-se, porém, manter o modelo base I uma vez que este capturou da forma esperada o efeito de inércia e apresentou resultados interessantes no caso da categoria de viajantes a negócios, como será discutido a seguir. No caso do modelo base II, pode-se também observar na tabela a estimativa do valor do parâmetro do ninho dos modos de transporte coletivo $\left(\mu_{\text {coletivo }}\right)$. Em alguns modelos, os valores das estatísticas t-Student são relativamente baixos, fato que pode decorrer da reduzida amostra disponível; esta consideração levou à manutenção das variáveis nos modelos, mesmo com menor significância, uma vez que os coeficientes apresentaram os sinais esperados.

É importante destacar que em geral não é possível comparar diretamente os valores absolutos dos coeficientes de modelos de estruturas distintas, como é o caso dos modelos base I e II, em função dos diferentes fatores de escala implícitos. Pode-se, porém, comparar a relação entre coeficientes ou trade-offs. A Tabela 5 apresenta duas estimativas de

trade-offs: o valor do tempo de viagem (VTV) e a importância relativa da margem de segurança (IMS). O VTV é obtido dividindo-se o coeficiente do custo pelo coeficiente do tempo de viagem; o IMS é calculado dividindo-se o coeficiente da margem de segurança pelo coeficiente do tempo.

Os itens a seguir discutem os resultados dos modelos obtidos a partir dos modelos base, incorporando interações que buscaram capturar variações sistemáticas de preferência. Embora tenham sido estimadas especificações incluindo interações com todos os atributos da função de utilidade, apenas as interações com o custo de viagem foram mais significativas. Os modelos com resultados mais satisfatórios são apresentados na Tabela 5. O modelo base I deu origem aos modelos 1.1 e 1.2 na tabela; os modelos 2.1 e 2.2 são derivados do modelo base II.

\subsubsection{Efeito do motivo de viagem}

Diversos autores concordam que viajantes a negócios a- 
Tabela 5. Modelos estimados

\begin{tabular}{|c|c|c|c|c|c|c|}
\hline \multirow[b]{2}{*}{ Variável } & \multicolumn{2}{|c|}{ Modelo Base I } & \multicolumn{2}{|c|}{ Modelo 1.1} & \multicolumn{2}{|c|}{ Modelo 1.2} \\
\hline & Estimativa & Teste- $t$ & Estimativa & Teste- $t$ & Estimativa & teste-t $t$ \\
\hline Custo & $(0,0134)$ & 1,41 & $(0,0200)$ & 1,74 & $(0,0407)$ & 2,73 \\
\hline Custo-Negócios & & & 0,0100 & 1,07 & & \\
\hline Custo-Renda & & & & & 0,0362 & 2,70 \\
\hline Tempo de Viagem & $(0,0078)$ & 1,16 & $(0,0078)$ & 1,16 & $(0,0078)$ & 1,14 \\
\hline Margem de Segurança & $(0,0165)$ & 1,85 & $(0,0166)$ & 1,85 & $(0,0169)$ & 1,85 \\
\hline Inércia & 0,3915 & 1,03 & 0,4045 & 1,05 & 0,4527 & 1,15 \\
\hline Sigma_Painel & 2,2727 & 3,60 & 2,3287 & 3,62 & 2,2804 & 3,64 \\
\hline Constante Táxi & 1,2899 & 1,39 & 1,3718 & 1,46 & 1,3769 & 1,47 \\
\hline Constante Táxi_Negócios & & & & & & \\
\hline Constante Trem & 2,1302 & 2,93 & 2,1750 & 2,93 & 2,2024 & 3,01 \\
\hline Constante Ônibus & 0,6202 & 0,98 & 0,6515 & 1,01 & 0,6357 & 1,00 \\
\hline$\mu_{\text {coletivo }}$ & & & & & & \\
\hline VTV* & 14,17 & & 18,93 & (1) & 41,61 & (3) \\
\hline VTV* & & & 9,47 & (2) & 4,64 & (4) \\
\hline IMS & 2,1 & & 2,1 & & 2,2 & \\
\hline Número de draws & 5000 & & 5000 & & 5000 & \\
\hline Máxima verossimilhança & $-180,3$ & & $-179,7$ & & $-175,0$ & \\
\hline$\rho^{2}$ ajustado & 0,371 & & 0,370 & & 0,386 & \\
\hline
\end{tabular}

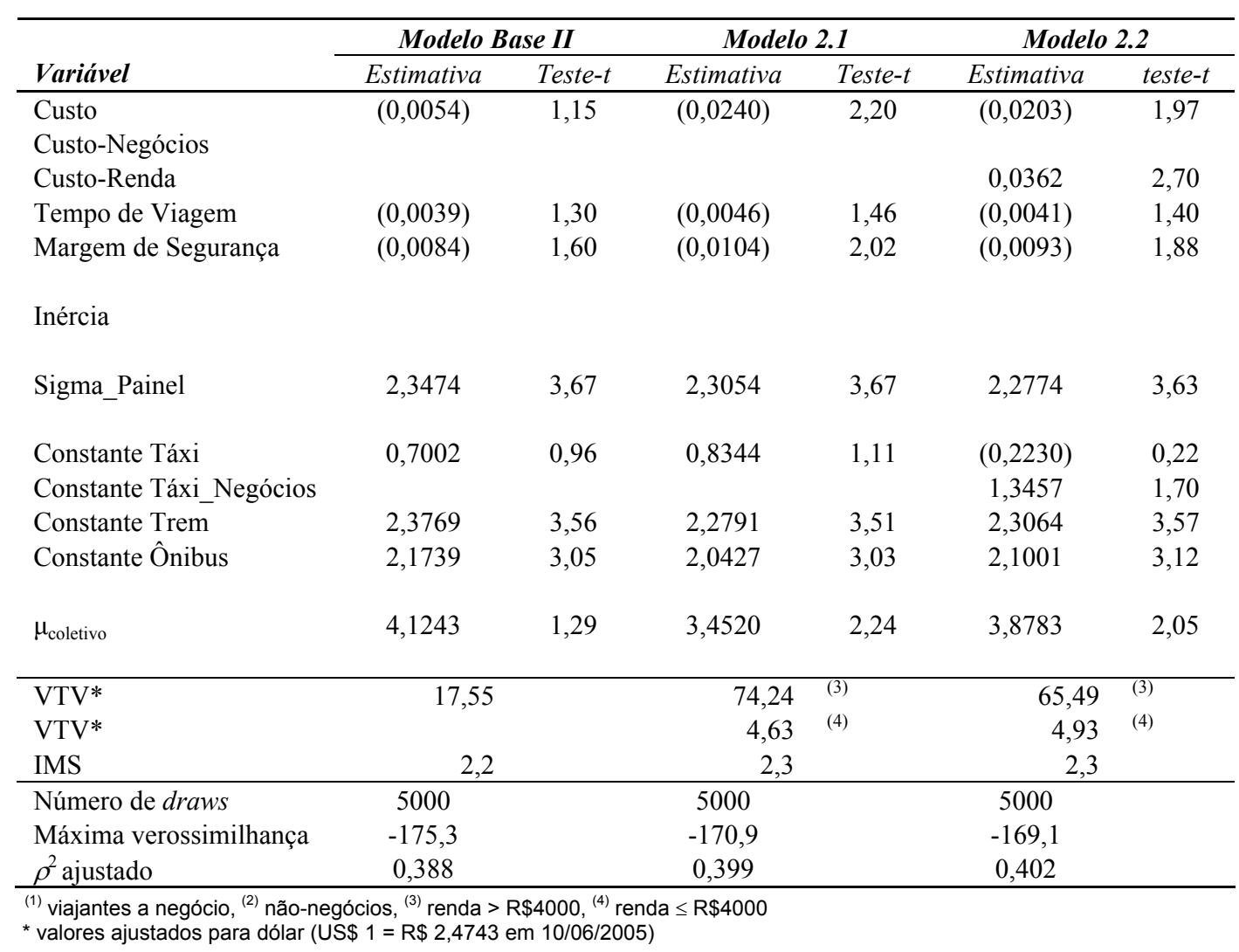

presentam valores do tempo até $80 \%$ mais altos do que viajantes por outros motivos (Pels et al. 2003, Tsamboulas e Nikoleris, 2008). Estes tendem a valorizar mais o tempo de viagem e menos o custo, seja porque não pagam por sua viagem (que pode ser paga pela empresa), seja porque o tempo gasto no acesso ao aeroporto pode ser considerado como tempo de trabalho desperdiçado. O modelo 1.1 incorpora uma variável binária - que representa o viajante a negócios - interagindo com o coeficiente de custo. Esta especifica- ção aumenta a significância do coeficiente de custo para a categoria de referência (não-negócios), porém, a significância da interação custo-negócios é baixa. O ajuste do modelo, indicado pelo valor de $\rho^{2}$ ajustado, decresce com a inclusão de mais uma variável. A interação custo-negócios utilizando o modelo base II não apresentou resultados significativos e não é apresentada na tabela. 


\subsubsection{Efeito da renda}

A renda individual declarada é uma variável que pode influenciar a escolha de modo. Bhat e Sardesai (2006) verificaram que indivíduos de alta renda parecem evitar modos coletivos de transporte, resultado que não se verificou neste estudo, considerando que a taxa de escolha de modos coletivos no experimento é muito similar para viajantes de alta e baixa renda. Por outro lado, conforme mostram os modelos 1.2 e 2.1 , o efeito da renda sobre o coeficiente do custo é significativo e reduz drasticamente a sensibilidade dos passageiros de alta renda ( $>$ R $\$ 4000 /$ mês em junho de 2005) em relação ao custo da viagem. A baixa sensibilidade dos viajantes aéreos com relação aos custos do acesso terrestre foi também observada por outros autores (Koster et al., 2010). Nota-se ainda que a inclusão da interação da variável indicativa de renda elevada com o custo da viagem aumentou a significância dos coeficientes dos dois modelos de forma geral, assim como melhorou o ajuste.

Com base nos resultados acima, foram testadas especificações que incluíam conjuntamente o efeito de renda e do motivo de viagem e suas interações com o coeficiente de custo; nesses casos, o efeito da renda dominou o motivo de viagem, que se tornou não significativo.

\subsubsection{Outros efeitos}

Diversas outras categorias da população amostral foram analisadas para verificar a existência de possíveis variações sistemáticas; a influência do gênero na escolha do modo e uma eventual preferência feminina pelos modos não coletivos, observada em alguns estudos, não pôde ser comprovada, tampouco a influência da frequência de viagens do viajante e suas interações com os atributos de escolha. Mais surpreendente foi não verificar efeitos significativos do dia da semana (sexta-feira x sábado), considerando as diferenças de tempo esperado de viagem e, principalmente, variabilidade do tempo de viagem.

Uma última especificação derivada do modelo base II é apresentada no modelo 2.2, e inclui a interação da constante específica do modo táxi com a variável binária indicativa do motivo de viagem, além da interação custo-renda já descrita (modelo 2.1). A ideia é a de que viajantes a negócios apresentem uma preferência pelo táxi não explicitamente considerada nas especificações acima. Por exemplo, é possível que a flexibilidade apresentada por este modo de transporte traga benefícios para o indivíduo que viaja desacompanhado (situação mais comum em viagens a negócios), que pode também não ter a disponibilidade de carona para o aeroporto. Além disso, em alguns casos, o viajante a negócios tem como origem de sua viagem terrestre o local de trabalho, tornando o táxi uma opção mais adequada. $\mathrm{O}$ modelo 2.2 apresenta o maior $\rho^{2}$ ajustado entre os modelos testados (passando de 0,388 no modelo base II para 0,402 ), além de apresentar uma melhora de significância nos coeficientes dos atributos e, consequentemente, nas estimativas de VTV e IMS.

\subsubsection{Valor do tempo de viagem (VTV) e importância da margem de segurança (IMS)}

O VTV apresentou variações interessantes entre os modelos apresentados na Tabela 5. Considerando os modelos base I e II, os valores do tempo não foram muito diferentes para o conjunto da amostra (cerca de US\$14 e US\$18, respectivamente). Viajantes a negócio revelaram um VTV cer- ca de 2 vezes maior do que viajantes por outros motivos (modelo 1.2). Koster et al. (2010) encontraram resultados semelhantes, embora os VTV calculados por estes sejam sensivelmente maiores (da ordem de grandeza de $90 € /$ hora para viajantes a negócio). Valores mais próximos a esses (entre US\$65/hora e US\$74/hora) foram encontrados para viajantes de alta renda nos modelos 2.1 e 2.2 (especificações que incluem o efeito da renda) e refletem a baixa sensibilidade dos viajantes de alta renda ao custo da viagem de acesso terrestre. Para viajantes no estrato de renda inferior a $\mathrm{R} \$ 4000$, os valores do tempo são bastante estáveis entre modelos (1.2, 2.1 e 2.2), ligeiramente inferiores a US $\$ 5 /$ hora.

O IMS, por outro lado, manteve-se bastante estável em todas as especificações testadas, aumentando nos modelos com melhor ajuste. O IMS calculado indica que os viajantes, quando desacompanhados, valorizam a margem de segurança cerca de duas vezes mais do que valorizam o tempo de viagem. A literatura apresenta uma vasta gama de valores para a confiabilidade de tempo de viagem, estimadas de acordo com cenários e ambientes específicos. De maneira geral, a maioria dos estudos concorda que a variabilidade do tempo de viagem é mais valorizada do que o tempo de viagem propriamente dito (ITS, 2008).

\section{CONCLUSÃO}

As características do acesso terrestre a um aeroporto afetam sua atratividade, e diversos autores têm incluído variáveis de acesso terrestre nos modelos de demanda de aeroportos, tais como tempo de viagem e custo. No entanto, os efeitos da variabilidade do tempo de viagem são raramente considerados nestes estudos, apesar de sua importância em uma viagem que requer um elevado grau de pontualidade.

Esse artigo utiliza os resultados de uma pesquisa de preferência declarada (PD) conduzida em 2005 para estimar a importância da confiabilidade do tempo de viagem na escolha de modo de transporte para o acesso terrestre a aeroportos. O aeroporto internacional André Franco Montoro, em São Paulo, foi utilizado como estudo de caso, devido a pouca confiabilidade no atual acesso terrestre rodoviário (por carro, táxi ou ônibus direto) e a proposta de se construir uma ligação expressa ferroviária com o centro da cidade. Foram coletados dados de viajantes a destinos internacionais. Os atributos utilizados para descrever o experimento de escolha PD foram custo da viagem, tempo de viagem esperado e confiabilidade do tempo de viagem, expressa através de uma margem de segurança, definida como o período de tempo alocado pelo indivíduo para chegar ao aeroporto no horário desejado. Uma análise preliminar dos dados mostrou uma forte preferência pelo trem, um modo que supostamente apresenta tempos de viagem mais confiáveis.

Os dados PD foram utilizados para a estimação de modelos de escolha discreta. Um modelo de componentes de erro e um modelo hierárquico misto, desenvolvidos em um trabalho anterior (Alves e Strambi, 2010), foram utilizados como base para a análise neste artigo. Em uma busca por variações sistemáticas de preferências, verificou-se a presença de interações significativas entre os atributos das alternativas e algumas categorias dos indivíduos na amostra. Mais especificamente, a renda individual e o motivo de viagem apresentaram interação significativa com o coeficiente de custo. Não foram encontradas diferenças significati- 
vas entre dias da semana (sexta e sábado), apesar destes apresentarem diferenças consideráveis em termos de tempo de viagem e variabilidade.

Embora os coeficientes dos atributos nos modelos não sejam sempre diretamente comparáveis devido a diferenças de escala, os trade-offs, calculados como razões entre coeficientes, são. Dois trade-offs foram calculados: o valor do tempo de viagem e o índice de importância da margem de segurança. Os resultados indicam que viajantes a negócios apresentam valor do tempo cerca de duas vezes maior que os viajantes por outros motivos, e viajantes de alta renda apresentam baixa sensibilidade ao custo, apresentando por vezes valores do tempo muito altos, fato também constatado por outros autores. Os modelos estimados indicam também que o táxi apresenta maior atratividade para os viajantes a negócio do que para viajantes por outros motivos, o que pode ser consequência da flexibilidade oferecida pelo táxi em relação às demais alternativas.

Embora calculados segundo cenários bastante diferentes, os estudos encontrados na literatura apresentam, em geral, valores da importância da confiabilidade maiores do que para o tempo de viagem. Em concordância com estes resultados, este estudo indica que a margem de segurança tem uma importância relativa de cerca de um pouco mais de duas vezes a do tempo de viagem médio. Este resultado mantém-se estável em todas as especificações testadas.

\section{AGRADECIMENTOS}

Os autores agradecem o apoio financeiro da CAPES e do CNPq. Juan de Dios Ortúzar, Elisabetta Cherchi e Sebastián Raveau foram de inestimável ajuda na estimação dos modelos.

\section{REFERÊNCIAS BIBLIOGRÁFICAS}

Alves, B. B. (2005) A importância da variabilidade do tempo de viagem no acesso terrestre a aeroportos: estudo de caso do Aeroporto Internacional André Franco Montoro. Dissertação de Mestrado. Escola Politécnica da Universidade de São Paulo, São Paulo.

Alves, B. B. e O. Strambi (2010) Travel time reliability on airport ground access. Proceedings of 12 th World Conference on Transport Research. WCTR, Lisboa.

Bates, J.; A. Cook; P. Jones e J. W. Polak (2001) The valuation of reliability for personal travel. Transportation Research E, v. 37, p. 191229.

Ben-Akiva, M.; T. Morikawa e F. Shiroishi (1991) Analysis of the reliability of preference ranking data. Journal of Business Research, v. 23, p. 253-268.

Bhat, C. R. e R. Sardesai (2006) The impact of stop-making and travel time reliability on commute mode choice. Transportation Re$\operatorname{search} B$, v. 40, p. 709-730.

Bierlaire, M. (2003) BIOGEME: a free package for the estimation of discrete choice models, Proceedings of the 3rd Swiss Transportation Research Conference, Ascona.

Bierlaire, M. (2008) An introduction to BIOGEME Version 1.7. Disponível em : $<$ http://biogeme.epfl.ch/>.

Brito, A. N. e O. Strambi (2007) Análise de características relacionadas à variação do valor do tempo de viagem de motoristas usando técnicas de preferência declarada. Transportes, v. 15, p. 50-57.

Brownstone, D. e K. A. Small (2005) Valuing time and reliability: assessing the evidence from road pricing demonstrations. Transportation Research A, v. 39, p. 279-293.

Hensher, D. A. e W. H. Greene (2003) The mixed Logit model: the state of practice. Transportation, v. 30, p. 133-176.

Hess, S.; J. M. Rose e J. Polak (2010) Non-trading, lexicographic and inconsistent behaviour in stated choice data. Transportation Research D, v. 15, p. 405-417.

Hollander, Y. (2006) Direct versus indirect models for the effects of unreliability. Transportation Research A, v. 40, p. 699-711.

Institute for Transport Studies (2008) Multimodal travel time variability Final Report. University of Leeds, Leeds.
Koster, P.; E. Kroes e E. T. Verhoef (2010) Travel time variability and airport Accessibility. Tinbergen Institute Discussion Paper TI 2010-061/3. Tinbergen Institute, Amsterdam.

Louviere, J. J.; D. A. Hensher e J. D. Swait (2000) Stated choice methods: analysis and application. Cambridge University Press, Cambridge.

Noland, R.B. e J. W. Polak (2002) Travel time variability: a review of theoretical and empirical issues. Transport Reviews, v. 22, p. 39-54.

Ortúzar, J.D. e L. G. Willumsen (2001) Modelling Transport (3rd.ed.) Wiley, Chichester.

Pells, S. (1987) The evaluation of reductions in travel time variability. Thesis (Ph.D). University of Leeds, Leeds.

Pels, E.; P. Nijkamp e P. Rietveld (2003) Access to and competition between airports: a case study for the San Francisco Bay area. Transportation Research A, v. 37, p. 71-83.

Swait, J. e J. Louviere (1993) The role of the scale parameter in the estimation and use of multinomial logit models. Journal of Marketing Research, v. 30, p. 305-314.

Tam, M. L.; W. H. K. Lam e H. P. Lo (2008) Modeling air passenger travel behavior on airport ground access mode choices. Transportmetrica, v. 4, p. 135-153.

Train, K. (2009) Discrete choice methods with simulation (2 ${ }^{\mathrm{a}}$ ed.). Cambridge University Press, Cambridge.

Transportation Research Board (2008) Airport ground access mode choice models. ACRP Synthesis 5. Transportation Research Board, Washington, D.C.

Tsamboulas, D. A. e A. Nikoleris (2008) Passengers' willingness to pay for airport ground access time savings. Transportation Research $A$, v. 42 , p. $1274-1282$

Vincent, M. (2008) Measurement valuation of public transport reliability. Land Transport New Zealand Research Report 339. Land Transport New Zealand, Wellington. 